\title{
O impacto da obra de J. B. Watson na psicologia norte-americana: Alguns parâmetros de comparação
}

\section{The impact of J. B. Watson's work on American Psychology: Some comparison parameters}

\author{
El impacto de la obra de J. B. Watson en la psicología \\ norteamericana: Algunos parámetros de comparación
}

\author{
Fernando Tavares Saraiva* \\ Universidade Federal do Pará - UFPA, Belém, Pará, Brasil \\ Marcus Bentes de Carvalho Neto** \\ Universidade Federal do Pará - UFPA, Belém, Pará, Brasil
}

Saulo de Freitas Araujo***

Universidade Federal de Juiz de Fora - UFJF, Juiz de Fora, Minas Gerais, Brasil

\begin{abstract}
RESUMO
O impacto da obra de J. B. Watson tem sido explorado na historiografia da psicologia. Neste âmbito, destacam-se estudos que utilizam como método a análise bibliométrica. Tais pesquisas, no entanto, não utilizam parâmetros comparativos que possam indicar de forma mais precisa o grau de impacto daquela obra. O presente estudo busca preencher tal lacuna por meio de uma análise bibliométrica comparativa das referências às obras de Watson e outros três relevantes psicólogos do período: Edward B. Titchener, Edward L. Thorndike e William James. A pesquisa foi realizada em cinco importantes periódicos norte-americanos da área, entre os anos 1903 e 1923 - uma década antes e uma década após a publicação do Manifesto Behaviorista. Os resultados permitem concluir que, embora não possa ser tomada propriamente como um marco revolucionário, a obra de Watson teve, na década posterior à publicação do Manifesto Behaviorista (1914-1923), um impacto próximo ao de Titchener e maior do que o de Thorndike, ainda que distante da influência exercida por James.
\end{abstract}

Palavras-chave: J. B. Watson, Behaviorismo, análise bibliométrica, História da Psicologia.

\begin{abstract}
The impact of J. B. Watson's work has been explored in the historiography of psychology. In this context, bibliometric analysis has been often used as a methodological strategy. However, such studies do not present comparative parameters that may indicate more precisely its degree of impact. The goal of this paper is to fill this gap, by making a comparative bibliometric analysis of citations to Watson and three other representative psychologists at that time: Edward B. Titchener, Edward L. Thorndike and William James. Five
\end{abstract}


important American journals were scrutinized for the period between 1903 and 1923, a decade before and a decade after the publication of Watson's Behaviorist Manifesto. The results suggest that, even if it cannot be taken as a revolutionary milestone, Watson's work had, in the first decade after the publication of the Behaviorist Manifesto (1914-1923), an impact similar to Titchener's and higher than Thorndike's, although still distant from James's influence.

Keywords: J. B. Watson, Behaviorism, bibliometric analysis, History of Psychology.

\section{RESUMEN}

El impacto de la obra de J. B. Watson ha sido investigado en la historiografía de la psicología. En este contexto, se destacan los estudios que utilizan el análisis bibliométrico como método. Sin embargo, estos estudios no utilizan parámetros comparativos que pueden indicar con mejor precisión el grado de impacto del autor. El objetivo de este estudio es llenar este vacío por medio de un análisis bibliométrico comparativo de las citas de las obras de Watson y de otros tres sicólogos importantes en este período: Edward B. Titchener, Edward Thorndike y William James. La búsqueda se realizó en cinco periódicos norteamericanos importantes entre los años 1903 y 1923, una década antes y una década después de la publicación del Manifiesto Conductista de Watson. Los resultados permiten concluir que, aún que no pueda ser tomada propiamente como un hito revolucionario, la obra de Watson tuvo, en la década después de la publicación del Manifiesto Conductista (1914-1923), un impacto próximo al de Titchener y más alto que el de Thorndike, aún que muy distante de la influencia ejercida por James.

Palabras clave: J. B. Watson, Conductismo, análisis bibliométrico, Historia de la Psicología.

Em 24 de fevereiro de 1913, John Broadus Watson (1878-1958), então um jovem professor na Johns Hopkins University, iniciou uma série de oito conferências na Columbia University, em Nova York, com o intuito de apresentar e promover uma nova visão da psicologia. A primeira destas conferências, intitulada Psychology as the behaviorist views it, foi publicada, no mesmo ano e sob o mesmo nome, no periódico Psychological Review e lançou as bases para o surgimento de um novo movimento: o Behaviorismo (Buckley, 1989; Cohen, 1979).

De fato, Watson não deixa dúvidas de que seu objetivo é fundar uma nova psicologia, com base em três pilares centrais. Em primeiro lugar, o alinhamento metodológico às ciências da natureza: "A psicologia como o behaviorista a vê é um ramo experimental, puramente objetivo, da ciência natural" (Watson, 1913, p. 158). Em segundo lugar, a demarcação de um novo objeto de estudo, o comportamento: "Ou a psicologia deve mudar o seu ponto de vista, de modo a incluir fatos do comportamento (...) ou então o comportamento deve ser isolado como parte de uma ciência inteiramente separada e independente" (p. 159). Finalmente, mas 
não menos importante, um novo ideal: "Seu objetivo teórico é a previsão e o controle do comportamento" (p. 158).

Posteriormente conhecido como "Manifesto Behaviorista", o texto revela a postura iconoclasta de Watson em relação à psicologia norteamericana de sua época, que, em geral, é compreendida em termos da disputa entre duas concepções rivais: o funcionalismo e o estruturalismo (p. ex., Hothersall, 2006; Goodwin, 2005). ${ }^{1}$

Esse caráter iconoclasta da obra e da figura de Watson tem sido amplamente reconhecido e aceito na literatura, sem grandes controvérsias. Há outro ponto sobre o Manifesto, contudo, que tem gerado muita discussão entre os historiadores da psicologia, a saber, qual foi seu impacto real na psicologia norte-americana da época?

E importante notar que, pelo menos desde o século 18, a procura de uma fórmula para o desenvolvimento de uma psicologia científica nos moldes das ciências naturais já era um elemento comum no imaginário dos mais variados tipos de psicólogos, ainda que eles frequentemente entendessem essa relação da psicologia com as ciências da natureza de forma diferente (p. ex., Araujo, 2016; Sturm, 2009). Por isso, a proposta de uma nova psicologia científica não seria suficiente para justificar o caráter inovador do Manifesto. No caso de Watson, o que estava em jogo era o alinhamento da psicologia à biologia, mais especificamente ao modelo experimental de pesquisas com animais. Mas também aqui, como observa Carrara (1998), diversos psicólogos já vinham adotando posicionamentos semelhantes aos de Watson, de modo que já existia certa disposição favorável à sua proposta. Na verdade, de acordo com o mesmo autor, - Manifesto representaria "a convergência de uma série de posições que ansiavam por essa mudança, mas não tinham encontrado o modo como operá-la. (...) Seu artigo foi exatamente ao encontro da expectativa presente no meio científico da época" (Carrara, 1998, p. 18).

Em que pese, porém, o cenário propício à recepção da proposta de Watson, isso parece não ter ocorrido de forma satisfatória, pelo menos nas proporções esperadas pelo próprio Watson, como ele reconhece na sua autobiografia (Watson, 1936/1950). Seja como for, a questão do real impacto tanto do Manifesto quanto de outras obras de Watson na psicologia norte-americana, apesar de ter recebido atenção na historiografia da psicologia, ainda permanece mal compreendida. Inicialmente, autores como Flugel (1933/1964), Boring (1950) e Herrnstein e Boring (1966) exaltaram a enorme repercussão da proposta de Watson e uma rápida adesão dos psicólogos a ela. De modo semelhante, os dois principais biógrafos de Watson insistiram no impacto quase instantâneo da proposta watsoniana. Cohen (1979) fala de "um sucesso imediato" (p. 73) e Buckley (1989) afirma que "a declaração de Watson provocou uma reação generalizada de psicólogos e cientistas sociais" (p. 78). 
Estudos mais recentes, porém, contestam a ideia de sua imediata e generalizada aceitação (Carvalho Neto et al., 2015; Coleman, 1988; García-Penagos, \& Malone, 2013; Marr, 2013; Samelson, 1981; Tortosa, Delgado, \& Garrído, 1991). Samelson (1981), por exemplo, por meio da análise de artigos publicados em periódicos relevantes da época, aponta que o Manifesto só teve um impacto significativo na psicologia norte-americana a partir do início da década de 1920, mas não nos primeiros anos após sua publicação.

Nessas discussões, a análise bibliométrica tem sido utilizada como um relevante recurso para a avaliação do impacto da obra em questão, gerando resultados que contestam a tese do grande impacto inicial dos escritos behavioristas de Watson. Tortosa et al. (1991), por exemplo, analisam as citações das obras de Watson em periódicos publicados durante dois períodos distintos: 1900-1945 e 1966-1985. Já Coleman (1988) estabelece uma comparação entre as referências às obras de Watson e Pavlov em um único periódico (Psychological Review). Todd (1994), por sua vez, analisa manuais e livros introdutórios de psicologia, buscando determinar o modo como estes apresentam alguns aspectos centrais da proposta do programa psicológico de Watson.

Seguindo essa tradição de estudos bibliométricos empreendidos sobre o tema, Carvalho Neto et al. (2015) conduziram uma investigação na qual buscaram avaliar o impacto da obra de Watson na psicologia norte-americana durante a primeira década seguinte à publicação do Manifesto. Como diferencial deste trabalho, os autores buscaram preencher uma lacuna metodológica observada nos estudos bibliométricos anteriores. De acordo com os autores, tais estudos em geral pecavam ou por não apresentarem uma amostra representativa da produção psicológica do período ou pela falta de clareza na apresentação do método e dos resultados obtidos.

Foram conduzidos, então, dois estudos bibliométricos. No Estudo 1, Carvalho Neto et al. (2015) buscaram especificamente avaliar a frequência de citações do Manifesto em quatro periódicos representativos da época (American Journal of Psychology, Psychological Review, Psychological Bulletin e Journal of Philosophy, Psychology and Scientific Methods/Journal of Philosophy). No Estudo 2, a busca foi ampliada para o nome e para a obra geral de Watson na mesma base de dados e no mesmo período. Os principais resultados revelaram que o Manifesto foi citado 20 vezes ao longo do período analisado, sendo $60 \%$ nos dois primeiros anos. Entretanto, o Manifestofoi apenas a terceira publicação mais citada de Watson em termos proporcionais. A obra mais referenciada foi o livro Psychology from the Standpoint of a Behaviorist (1919), seguido pelo livro Behavior: An Introduction to Comparative Psychology (1914).

$\mathrm{Na}$ pesquisa empreendida por Carvalho Neto et al. (2015), cuja ênfase recaiu sobre a questão do refinamento de um método 
bibliométrico para aferir o impacto da obra de Watson, os autores apresentaram algumas limitações do estudo. A principal delas foi a ausência de parâmetros de comparação claros e objetivos que pudessem ser utilizados como base para estimar o impacto de uma obra. O que significaria, por exemplo, ter uma obra citada 20 vezes ao longo de 10 anos? Esse número seria grande ou pequeno? Ainda que o método tenha permitido acompanhar mais direta e facilmente a frequência de referências a Watson e sua obra ao longo do tempo, identificando se houve aumento, redução ou manutenção dos índices, não foi possível avaliar seu impacto em termos comparativos com a área como um todo, levando em consideração o contexto mais amplo da produção psicológica da época.

Os autores sugerem, então, duas estratégias adicionais que poderiam ser utilizadas para aprofundar a compreensão dos dados obtidos: (a) uma análise qualitativa das citações, que pudesse verificar como estas apresentam e debatem a obra; e (b) uma avaliação quantitativa de outros autores representativos do período, que pudesse servir como parâmetro externo de comparação.

Dando continuidade ao estudo de Carvalho Neto et al. (2015), o objetivo do presente trabalho foi exatamente implementar essa segunda estratégia metodológica e fornecer informações adicionais para a mensuração do impacto da obra de Watson. Nesse caso, foram escolhidos para a comparação os seguintes nomes: Edward B. Titchener (1867-1927), Edward L. Thorndike (1874-1949) e William James (1842-1910). A justificativa para essa escolha é dupla. Em primeiro lugar, os referidos autores figuram entre os mais eminentes representantes da psicologia norte-americana nas primeiras décadas do século XX (Catania, 1999; Ferreri, 2006; Haggbloom et al., 2002; Heidbreder, 1981/1933; Knight, 1953). Além disso, eles foram citados nominalmente por Watson em sua crítica àquilo que entendia como "velha psicologia". No caso específico de Titchener, vale ainda ressaltar que ele foi o autor de uma das primeiras respostas acadêmicas ao Manifesto, na qual discute em detalhe a proposta watsoniana (Titchener, 1914). Espera-se, assim, fornecer uma base inicial para uma futura comparação entre Watson e outros importantes autores do período.

\section{MÉTODO}

Foi realizada uma análise bibliométrica das citações das obras de Watson, James, Thorndike e Titchener, de acordo com as diretrizes metodológicas estabelecidas por Brǒzek para a historiografia da psicologia (Brǒzek, 1969a, 1969b, 1970, 1972, 1980). A ideia subjacente à utilização desse método é que a análise quantitativa de referências representa um importante fator, embora não certamente 
o único, na mensuração do impacto científico de um autor ou obra, como também defendem Carpintero e Peiro (1983).

O período escolhido para a análise foi uma década antes e uma década depois da publicação do Manifesto (1903-1923). Embora essa escolha possa parecer arbitrária, o que é comum em relação aos recortes temporais estabelecidos em pesquisas históricas, ela pode ser justificada em termos de suas vantagens práticas. Primeiro, 0 presente recorte permite uma comparação direta com o estudo anterior de Carvalho Neto et al. (2015), do qual esse trabalho é uma continuação. Segundo, ele permite igualmente um confronto com a análise de Samelson (1981), que representa a primeira tentativa de examinar o problema de maneira mais sistemática. Em ambos os casos, os autores adotaram o mesmo recorte de uma década. Finalmente, esse recorte facilita a gradual ampliação do escopo do presente projeto de pesquisa, cuja previsão é avançar década a década.

Foram selecionados cinco periódicos para a busca e mensuração das citações: Psychological Bulletin; Psychological Review; The American Journal of Psychology; Journal of Philosophy, Psychology and Scientific Methods - que posteriormente mudou o nome para Journal of Philosophy -; e Proceedings of the American Philosophical Society. Esses periódicos figuram entre os mais tradicionais da época, sendo amplamente utilizados pela comunidade psicológica norte-americana no início do século XX (Green \& Feinerer, 2015; Green, Feinerer, \& Burman, 2015a, 2015b; Osier \& Wozniak, 1984). Além disso, uma busca na base de dados PsycINFO da American Psychological Association

(http://www.apa.org/pubs/databases/psycinfo/index.aspx), na qual estão registradas as publicações psicológicas desde o início do século $X X$, revela que quatro dos cinco periódicos selecionados figuram entre os 10 mais prolíficos da época.

A pesquisa foi realizada em duas bases de dados eletrônicas: a Psycnet, pertencente à American Psychological Association (APA), onde estão indexados os periódicos Psychological Bulletin (PB: 1904...) e Psychological Review (PR: 1894-...); e a JSTOR, na qual estão indexados os periódicos The American Journal of Psychology (AJP: 1887-...), Journal of Philosophy, Psychology and Scientific Methods (JPPSM: 1904-1920)/Journal of Philosophy (JPh: 1921-...) e Proceedings of the American Philosophical Society (PAPS: 18382012). Em cada um desses periódicos, foram realizadas oito buscas com os termos "Watson", "J. B. W.", "Titchener", "E. B. T.", "Thorndike", "E. L. T.", "James" e "W. J.", abrangendo o período entre 1903 e 1923. As formas abreviadas dos nomes dos autores foram utilizadas como palavras-chave porque, nesta época, era comum assinarem desta forma alguns de seus textos, particularmente as resenhas. 
Uma vez selecionados os artigos, estes eram salvos em computador para posterior análise. Em cada um deles era, então, realizada uma busca para identificar o trecho do texto no qual a palavra-chave aparecia. Caso a palavra-chave realmente fizesse referência a um dos autores escolhidos, o artigo era computado como parte da pesquisa e categorizado de acordo com a palavra-chave que continha. ${ }^{2}$ Neste caso, foram utilizadas quatro categorias: "Watson + J. B. W.", "Titchener + E. B. T.", "Thorndike + E. L. T." e "James + W. J.". Para os fins da presente investigação foram considerados apenas artigos, discussões (que incluem réplicas, comentários, etc.) e resenhas de livros nos quais fosse possível identificar a autoria. ${ }^{3}$ Foram desconsiderados tanto os demais formatos - editoriais, correspondências, notas, anúncios, notícias, coletâneas de resumos, etc. - quanto artigos, resenhas ou discussões de autoria dos próprios autores em suas respectivas categorias, a fim de evitar que a autorreferência fosse computada como impacto. Após a exclusão desse material, foi utilizado como critério de inclusão apenas a presença das já referidas palavras-chave, as quais determinavam também a categoria em que os trabalhos seriam analisados.

Após a coleta de dados, a análise foi realizada em função das seguintes medidas:

(1) Total de citações: faz referência à quantidade total de publicações que citam os nomes de Watson, Titchener, Thorndike ou James pelo menos uma vez ao longo do texto;

(2) Taxa de citação: refere-se à média aritmética simples de publicações com citações do nome de cada um dos referidos teóricos - de acordo com a categoria que está sendo analisada - em cada ano (total de publicações com referências ao autor no período em questão dividido pela quantidade de anos);

(3) Percentual de citação: percentual equivalente à quantidade de artigos, resenhas e discussões que citam Watson, Titchener, Thorndike ou James - de acordo com a categoria que está sendo analisada - dividido pela quantidade total de artigos, resenhas e discussões publicada nos cinco periódicos durante todo o período analisado (1903-1923). Para a análise desta medida, em cada categoria foram excluídas do universo total de publicações as obras de autoria do autor em questão. No caso, por exemplo, da categoria equivalente às citações do nome de Titchener, eram excluídas as obras de autoria de Titchener, mas eram mantidas no total de materiais publicados no período aquelas de autoria dos outros teóricos (Watson, Thorndike ou James). Para um maior detalhamento de tais medidas, ver Carvalho Neto et al. (2015). 


\section{RESULTADOS E DISCUSSÃO}

Seguindo o estudo original de Carvalho Neto et al. (2015), foram adotadas três medidas: (a) total de citações; (b) taxa de citação; (c) percentual de citação. A primeira seria o valor absoluto de citações; a segunda corresponderia ao valor absoluto dividido por ano; e a terceira seria o percentual de citações de um autor dentro do universo total de publicações estudadas.

Durante o período investigado (1903-1923), foram identificadas, nos cinco periódicos pesquisados, 221 publicações que citam Watson, 392 que citam Titchener, 273 que citam Thorndike e 932 que citam James. As taxas de citação obtidas (total de citações/ano) foram: 10,52 publicações que citam Watson por ano; 18,66 publicações que citam Titchener por ano; 13 publicações que citam Thorndike por ano; e 44,38 publicações que citam James por ano. Já no que se refere ao percentual de citação, os resultados foram os seguintes: Watson foi citado em $3,42 \%$ das obras examinadas; Titchener foi citado em $6,05 \%$ delas; Thorndike foi citado em 4,22\%; e James foi citado em $14,42 \%$ das obras examinadas. 
Tabela 1

Medidas das publicações que citam Watson, Titchener, Thorndike e James no periodo 1903-1923.

\begin{tabular}{|c|c|c|c|c|}
\hline \multicolumn{5}{|c|}{ Medida 1: Total de citaçôes } \\
\hline \multirow{2}{*}{ Período } & \multicolumn{4}{|c|}{ Autor } \\
\hline & "Watson" & "Titchener" & "Thorndike" & "James" \\
\hline $1903-1912$ & 26 & 163 & 95 & 513 \\
\hline 1913 & 12 & 24 & 17 & 36 \\
\hline $1914-1923$ & 183 & 205 & 161 & 383 \\
\hline $1903-1923$ & 221 & 392 & 273 & 932 \\
\hline \multicolumn{5}{|c|}{ Medida 2: Taxa de citação } \\
\hline \multirow{2}{*}{ Período } & \multicolumn{4}{|l|}{ Autor } \\
\hline & "Watson" & "Titchener" & "Thorndike" & "James" \\
\hline $1903-1912$ & 2,6 & 16,3 & 9,5 & 51,3 \\
\hline 1913 & 12 & 24 & 17 & 36 \\
\hline $1914-1923$ & 18,3 & 20,5 & 16,1 & 38,3 \\
\hline $1903-1923$ & 10,52 & 18,66 & 13 & 44,38 \\
\hline \multicolumn{5}{|c|}{ Medida 3: Percentual de citação } \\
\hline \multirow{2}{*}{ Período } & \multicolumn{4}{|c|}{ Autor } \\
\hline & "Watson" & "Titchener" & "Thorndike" & "James" \\
\hline $1903-1912$ & $0,79 \%$ & $5,03 \%$ & $2,90 \%$ & $15,71 \%$ \\
\hline 1913 & $3,52 \%$ & $7,05 \%$ & $5 \%$ & $10,58 \%$ \\
\hline $1914-1923$ & $6,40 \%$ & $7,23 \%$ & $5,65 \%$ & $13,38 \%$ \\
\hline $1903-1923$ & $3,42 \%$ & $6,11 \%$ & $4,22 \%$ & $14,42 \%$ \\
\hline
\end{tabular}

Medidas referentes ao número de publicações que citam Watson, Titchener, Thorndike e James, no conjunto dos cinco periódicos selecionados ("AJP, "PB", "PR", "JPPSM"/"JPh" e "PAPS"), divididas em 4 recortes cronológicos (uma década antes da publicaçăo do Manifesto Behaviorista, no ano de sua publicação, uma década após sua publicação e durante todo o período pesquisado).

A Tabela 1 apresenta as medidas das obras que citam cada autor ao longo de quatro recortes cronológicos: 1) a década anterior à publicação do Manifesto Behaviorista (1903-1912); 2) o ano de publicação desta obra, $1913 ; 3)$ a década posterior à sua publicação (1914-1923); e 4) todo o período contemplado pelos três recortes anteriores (1903-1923). Optou-se por adotar a publicação do Manifesto Behaviorista como referencial para tais recortes cronológicos devido à possibilidade de ele representar um marco na obra de Watson e na própria história da psicologia norte-americana.

Os dados apresentados foram analisados a partir de dois níveis, sendo um deles interno à obra de Watson - onde se buscou avaliar a mudança na quantidade de referências a Watson, tendo como comparação a sua própria obra - e outro externo, no qual se buscou parâmetros para aferir o alcance de sua obra a partir da comparação com o impacto da obra de outros autores.

No que se refere ao primeiro nível de análise, é possível demarcar claramente o quanto o nome de Watson passa a exercer influência 
após a publicação do artigo de 1913: empregando-se o cálculo da média aritmética referente às publicações que citam Watson na década anterior a 1913 (1903-1912), obtém-se a quantidade de 2,6 publicações por ano, enquanto que na década posterior a 1913 (1914-1923) este valor chega a 18,3 publicações por ano. O aumento no percentual de citação de sua obra ao longo das duas décadas também corrobora tal aumento de influência: na década anterior a 1913 , era equivalente a $0,79 \%$, passando a $6,40 \%$ na década seguinte. Assim, a partir desses dados, torna-se possível indicar 0 impacto da publicação do Manifesto Behaviorista no contexto da carreira acadêmica de Watson.

Um segundo nível de análise diz respeito à comparação entre as publicações que citam Watson e aquelas que citam os demais autores (Titchener, Thorndike e James). Como observado na Tabela 1, a partir da análise do total de referências feitas a cada autor em todo o período indicado (1903-1923), James e Titchener recebem um número consideravelmente maior de citações (932 e 392 obras, respectivamente), se comparados a Watson (221 obras). No entanto, quando o foco é apenas no período posterior a 1913 - ou seja, entre 1914 e 1923 -, a diferença entre Titchener (205 obras) e Watson (183 obras) diminui sensivelmente. 
Figura 1

Medidas das publicações que citam Watson, Titchener, Thorndike e James no periodo 1903-1923.
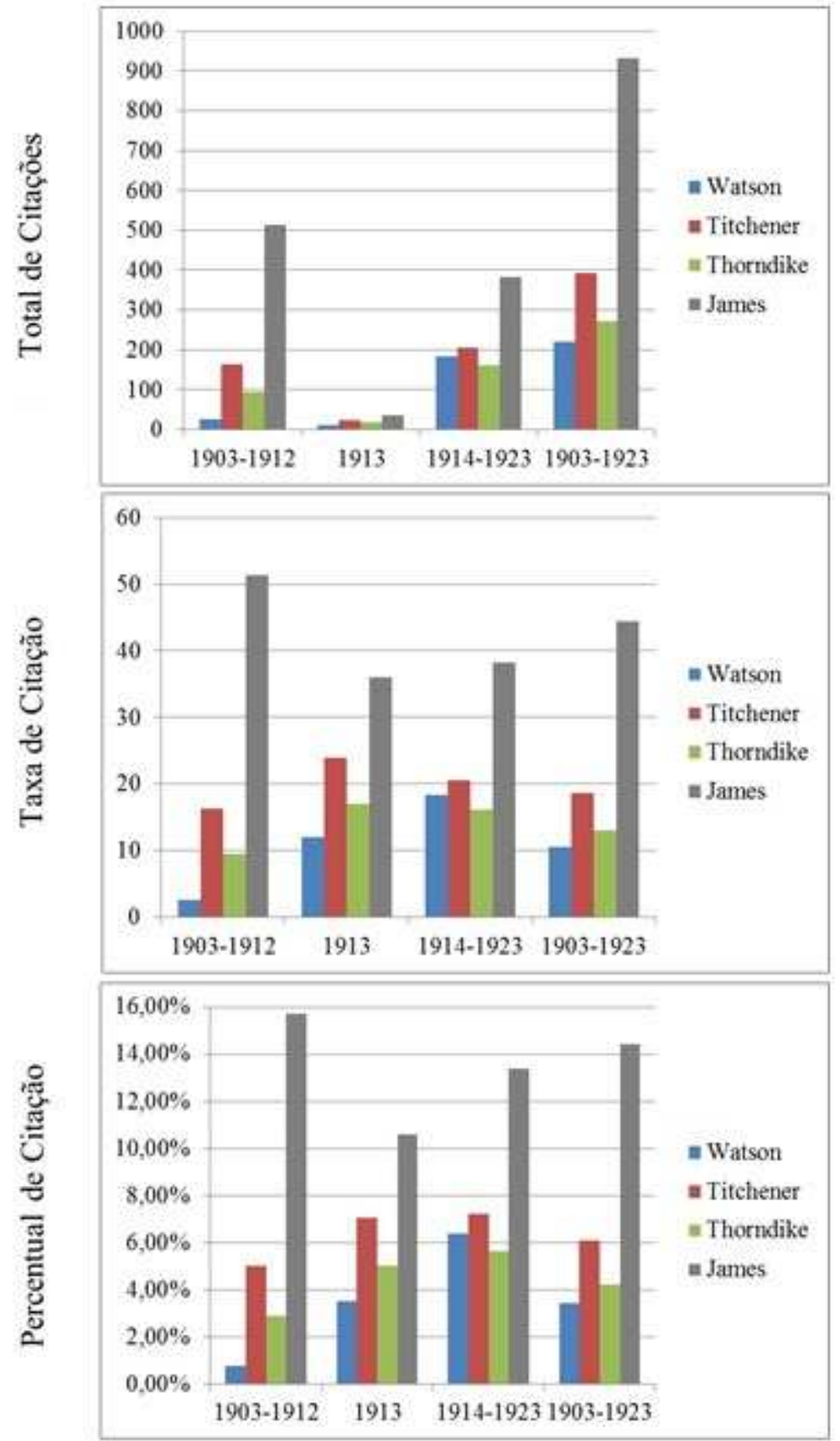

Periodo

Medidas referentes ao número de publicações que citam Watson, Titchener, Thorndike e James, no conjunto dos cinco periódicos selecionados ("AJP, "PB", "PR", "JPPSM"/"JPh" e "PAPS"), divididas em 4 recortes cronológicos (uma década antes da publicação do Manifesto Behaviorista, no ano de sua publicação, uma década após sua publicaçăo e durante todo o período pesquisado). 
A comparação das taxas de citação - como apresentado na Figura 1 permite analisar o número de citações das obras dos autores ao longo dos recortes cronológicos já apontados anteriormente, fornecendo uma medida mais refinada em relação à simples quantidade total de citações. A análise desta medida sugere outro interessante resultado: Watson seria o único dos autores incluídos neste estudo a apresentar um aumento na taxa de citação de suas obras durante os três períodos históricos consecutivos aqui analisados. Antes de 1913, Watson era citado em uma média de 2,6 publicações por ano; em 1913 passa a ser citado em 12 publicações e, por fim, alcança 18,3 publicações por ano no período entre 1914 e 1923 . No que se refere aos demais autores, tanto Titchener quanto Thorndike, apesar de também terem um aumento na taxa de citação de suas obras na passagem do primeiro para o segundo período - no caso de Titchener, de 16,3 publicações por ano para 24 publicações por ano; no de Thorndike, de 9,5 publicações por ano para 17 publicações por ano -, tiveram queda nessa taxa no último período da análise: 20,4 publicações por ano para Titchener e 16,1 publicações por ano para Thorndike. Em relação a James, também houve oscilação nessa taxa. No entanto, isso ocorreu em períodos distintos: ela passou de 51,3 publicações por ano na década anterior a 1913 para 36 publicações em 1913 , mantendo um valor próximo $(38,3)$ na década posterior. Por fim, adotando-se o percentual de citações como uma terceira medida, também representada na Figura 1, que permite a análise da quantidade de referências a cada autor dentro de um universo possível de publicações, pode-se perceber, tomando como parâmetro de comparação a década seguinte à publicação do Manifesto Behaviorista, que Titchener apresenta um percentual de 7,23\%, ao passo que Watson chega a $6,40 \%$. A proximidade entre os dados de ambos, neste caso, é considerável: inferior a $1 \%$. Se adotarmos as referências a Thorndike, os dados sugerem um aumento da influência de Watson neste período: antes de 1913, o percentual de referências a Watson era $0,79 \%$, enquanto o de Thorndike chegava a 2,90\%; já após 1913, apesar de haver um aumento no percentual de ambos os autores, o percentual de Watson $(6,40 \%)$ ultrapassa o de Thorndike $(5,65 \%)$.

Diante de tais resultados, algumas conclusões apresentadas anteriormente merecem discussão. No que se refere, por exemplo, à suposta rápida e generalizada adesão ao Behaviorismo a partir da publicação do Manifesto, como sugerido por Boring (1950) e outros autores, os resultados do presente estudo não sustentam tal interpretação. Apesar de demonstrar um considerável aumento na visibilidade de sua obra após 1913, próxima à de Titchener e maior que Thorndike, as citações das obras de Watson não apresentam maiores frequências do que as de Titchener e James, dois grandes nomes da psicologia norte-americana na época. Embora uma análise 
mais apressada, que leve em conta apenas as medidas internas à obra de Watson, possa sugerir uma rápida adesão à sua proposta, a introdução de novos parâmetros comparativos, externos à obra de Watson, refuta a tese de que esta última tenha provocado uma adesão generalizada e uma revolução imediata, uma vez que a quantidade de referências aos outros autores ou é maior (Titchener e James) ou apenas ligeiramente menor (Thorndike) do que as referências a Watson. Neste sentido, os resultados do presente estudo parecem reforçar a tese defendida por Carvalho Neto et al. (2015), segundo a qual a atitude dos psicólogos em relação ao Behaviorismo de Watson seria um ponto intermediário entre "a completa adesão" e a "pura indiferença".

Particularmente em relação à comparação com o estruturalismo de Titchener, poder-se-ia contestar que, no período posterior à publicação do Manifesto, ele já não representava mais uma posição tão hegemônica na psicologia, possuindo como defensores basicamente os pesquisadores ligados ao seu laboratório. No entanto, os resultados desta investigação revelam que, após a publicação do Manifesto Behaviorista, a influência da obra de Titchener nas publicações em psicologia é muito semelhante à do período imediatamente anterior. Portanto, não é possível inferir dos resultados aqui apresentados um declínio evidente do estruturalismo de Titchener a partir de 1913. É possível inferir, contudo, uma elevação da influência da proposta watsoniana, que chega a um patamar próximo àquele da psicologia titcheneriana.

Uma outra tese que também seria interessante retomar à luz dos resultados aqui obtidos é a de Tortosa et al. (1991), segundo a qual a posição de Watson nunca teria sido hegemônica na Psicologia e, deste ponto de vista, a sua proposta behaviorista não poderia representar uma ruptura paradigmática com o que era produzido em psicologia à época. Os resultados da presente investigação corroboram esta tese, uma vez que as referências ao pensamento de Titchener e James continuam a permear a produção científica em psicologia mesmo após a apresentação e divulgação inicial do Behaviorismo, tanto em 1913 quanto ao longo de sua primeira década de existência.

Por fim, a análise dos dados sugere que, após 1913, ainda que aparentemente distante do alcance das ideias de James, a nova proposta de Watson passou a ter uma influência próxima àquela exercida pelo estruturalismo de Titchener e maior do que 0 associacionismo de Thorndike. 


\section{CONSIDERAÇÕES FINAIS}

O presente estudo permite concluir duas coisas. Em primeiro lugar, é difícil sustentar a tese de que o Manifesto Behaviorista representa um marco revolucionário, em qualquer um dos sentidos tradicionais que o termo 'revolução' possui na literatura especializada. Em segundo lugar, contudo, é possível afirmar que a obra de Watson, ainda que distante do alcance da obra de James, passou a exercer uma influência próxima às produções de Titchener e Thorndike na primeira década após a publicação do Manifesto.

É importante apontar, porém, uma limitação da presente investigação, que poderia prejudicar a interpretação geral dos resultados. Não foram realizadas a categorização e a análise das obras citadas de cada autor e do contexto de cada citação. A introdução destes novos parâmetros, qualitativos, poderia ser útil em dois sentidos. Primeiro, várias referências a James e Titchener podem indicar muito mais uma menção a livros de introdução geral à psicologia do que uma filiação teórica ao seu pensamento. Além disso, no caso de James, é preciso levar em consideração o fato de que sua obra oscila entre a filosofia e a psicologia, de modo que seria interessante separar essas duas dimensões de sua influência, no intuito de corrigir uma eventual distorção deste estudo. Afinal, é possível que a expressiva quantidade de referências a James não corresponda à influência real de sua psicologia.

Os resultados alcançados sugerem, assim, a necessidade de se estabelecerem novos parâmetros de comparação, que permitam chegar a um maior refinamento da análise e, consequentemente, a um dimensionamento mais preciso do impacto da obra de Watson. Neste sentido, as sugestões de Carvalho Neto et al. (2015) quanto à necessidade de estudos longitudinais e da qualificação dos dados quantitativos coletados parecem adquirir maior pertinência, sugerindo, para além de uma análise bibliométrica das citações, um estudo qualitativo das mesmas.

\section{Referências}

Araujo, S. F. (2016). Wundt and the philosophical foundations of psychology: A reappraisal. New York: Springer.

Boring, E. G. (1950). A History of experimental psychology. New York: Appleton Century-Crofts.

Brǒzek, J. (1969a). History of psychology: Diversity of approaches and uses. Transactions of the New York Academy of Sciences, 31(2), 115-127.

Brǒzek, J. (1969b). Spectrum of Soviet psychology: 1968 model. American Psychologist, 24(10), 944-946. 
Brǒzek, J. (1970). Citation "longevity" as criterion of significance: "F. C. Donders (1986) and the timing of mental operations". Proceedings of the 78th Annual Convention of the American Psychological Association, 787-788.

Brǒzek, J. (1972). Quantitative explorations in the history of psychology in Yugoslavia: translations. Psychological Reports, 31(2), 397-398.

Brǒzek, J. (1980). The echoes of Wundt's work in the United States, 1887-1977: A quantitative citation analysis. Psychological Research, 42(1), 103-107.

Buckley, K. W. (1989). Mechanical man: John B. Watson and the beginnings of behaviorism. New York: Guilford Press.

Carrara, K. (1998). Behaviorismo radical: Crítica e metacrítica. Marília: UNESP.

Carvalho Neto, M. B., Araujo, S. F., \& Silva, E. G. (2015). O impacto da obra de J. B. Watson na psicologia norte-americana: Uma análise bibliométrica (1913-1923). Acta Comportamentalia, 23(4), 437-449.

Carpintero, H., \& Peiro, J. M. (1983). The significance of the bibliometric methodologyto the studies of the history of psychology. Revista de Historia de la Psicología, 4(1), 21-32.

Catania, A. C. (1999). Thorndike's legacy: Learning, selection, and the law of effect. Journal of the Experimental Analysis of Behavior, 72, 425-428.

Cohen, D. (1979). J. B. Watson: The founder of behaviourism. London: Routlege \& Kegan Paul.

Coleman, S. R. (1988). Assessing Pavlov's impact on the American conditioning enterprise. Pavlovian Journal of Biological Science, 23, 102-106.

Ferreri, A. M. (2006). The contribution of William James to the origins of "scientific" psychology. Physis, 43(1-2), 373-85.

Flugel, J. C. (1964). A hundred years of psychology. London: Methuen. (Trabalho original publicado em 1933).

García-Penagos, A., \& Malone, J. C. (2013). From Watson's 1913 manifesto to complex human behavior. Mexican Journal of Behavior Analysis, 39(2), 135-154.

Goodwin, C. J. (2005). História da psicologia moderna. São Paulo: Cultrix.

Green, C. \& Feinerer, I. (2015). The Evolution of The American Journal of Psychology 1, 1887-1903: A Network Investigation. The American Journal of Psychology, 128(3), 387-401.

Green, C., Feinerer, I., \& Burman, J. (2013). Beyond the schools of psychology 1: A digital analysis of Psychological Review, 18941903. Journal of the History of the Behavioral Sciences, 49(2), 167-189. 
Green, C., Feinerer, I., \& Burman, J. (2014). Beyond the schools of psychology 2: A digital analysis of Psychological Review, 19041923. Journal of the History of the Behavioral Sciences, 50(3), 249-279.

Green, C., Feinerer, I., \& Burman, J. (2015a). Searching for the structure of early American psychology: Networking Psychological Review, 1894-1908. History of Psychology, 18(1), 15-31.

Green, C., Feinerer, I., \& Burman, J. (2015b). Searching for the structure of early American psychology: Networking Psychological Review, 1909-1923. History of Psychology, 18(2), 196-204.

Haggbloom, S. J. et al. (2002). The 100 most eminent psychologists of the 20th century. Review of General Psychology, 6(2), 139152.

Heidbreder, E. (1981). Psicologias do século XX. São Paulo: Mestre Jou. (Trabalho original publicado em 1933).

Herrnstein, R. J., \& Boring, E. C. (Eds.) (1966). A sourcebook in the history of psychology. Cambridge: Harvard University Press.

Hothersall, D. (2006). História da psicologia. São Paulo: McGraw-Hill. Knight, M. (1953). The permanent contribution of William James to psychology. British Journal of Educational Psychology, 23(2), 77-86.

Marr, M. J. (2013). "It is not elementary, my dear Watson": The strange legacy of the Behaviorist Manifesto. Mexican Journal of Behavior Analysis, 39(2), 34-47.

Osier, D. V., \& Wozniak, R. H. (Eds.) (1984). A century of serial publications in psychology, 1850-1950: An international bibliography. Millwood, NY: Kraus.

Samelson, F. (1981). Struggle for a scientific authority: The reception of Watson's behaviorism, 1913-1920. Journal of the History of the Behavioral Sciences, 17, 399-425.

Sturm, T. (2009). Kant und die Wissenschaften vom Menschen. Paderborn: Mentis Verlag.

Titchener, E. B. (1914). On "Psychology as the behaviorist views it". Proceedings of the American Philosophical Society, 53(213), 117.

Todd, J. T. (1994). What psychology has to say about J. B. Watson: Classical behaviorism in psychology textbooks, 1920-1989. In J. T. Todd \& E. K. Morris (Eds.), Modern perspectives on John B. Watson and classical behaviorism (pp. 75-107). Westport: Greenwood Press.

Tortosa, F., Delgado, E. P., \& Garrído, A. P. (1991). La nueva imagen de John Broadus Watson en la historiografía contemporánea. Anuario de Psicología, 51, 67-87. 
Watson, J. B. (1913). Psychology as the behaviorist views it. Psychological Review, 20(2), 158-177.

Watson, J. B. (1950). John Broadus Watson. In C. Murchison (Ed.), A history of psychology in autobiography, volume 3 (pp. 271281). Worcester: Clark University Press. (Trabalho original publicado em 1936).

Woodworth, R. (1931). Contemporary schools of psychology. New York: Ronald Press.

\section{Endereço para correspondência \\ Fernando Tavares Saraiva}

Universidade Federal do Pará

Rua Augusto Corrêa, 1, Campus Universitário do Guamá, CEP 66075-110, Belém PA, Brasil

Endereço eletrônico: fernandotavaressaraiva@hotmail.com

\section{Marcus Bentes de Carvalho Neto}

Universidade Federal do Pará

Rua Augusto Corrêa, 1, Campus Universitário do Guamá, CEP 66075-110, Belém PA, Brasil

Endereço eletrônico: marcusbentesufpa@gmail.com

\section{Saulo de Freitas Araujo}

Universidade Federal de Juiz de Fora

Departamento de Psicologia, Campus Universitário, CEP 36036-900, Juiz de Fora MG, Brasil

Endereço eletrônico: saulo.araujo@ufjf.edu.br

Recebido em: 05/06/2016

Reformulado em: 28/09/2016

Aceito para publicação em: 27/11/2016

\section{Notas}

O presente artigo foi realizado com auxílio de bolsa de mestrado CAPES.

${ }^{1}$ Apesar de suas vantagens didáticas, essa narrativa historiográfica tradicional com base em escolas - introduzida por Woodworth (1931) e Heidbreder (1933/1981) apresenta um quadro geral muito simplificado do desenvolvimento da psicologia, como alguns estudos recentes têm mostrado (p. ex., Green, Feinerer, \& Burman, 2013, 2014, 2015). O problema central é que "muita coisa importante é deixada de fora, como as diversas tradições de pesquisa psicológica que não pertenceram historicamente a nenhuma das escolas" (Carvalho Neto, Araujo, \& Silva, 2015, p. 438).

${ }^{2}$ Nos casos específicos de Watson e James, houve grande ocorrência de citações de outros autores com o mesmo sobrenome.

3 É comum, no período analisado, a ocorrência de resenhas identificadas como "autoria desconhecida", as quais não foram consideradas como dados da presente pesquisa.

* Mestrando do Programa de Pós Graduação em Teoria e Pesquisa do Comportamento da UFPA.

** Doutor em Psicologia Experimental pela USP. Docente do Programa de Pós Graduação em Teoria e Pesquisa do Comportamento da UFPA.

*** Doutor em Filosofia pela UNICAMP/Universität Leipzig. Docente do Programa de Pós Graduação em Psicologia da UFJF. 